\title{
Indicadores de sensibilidad de una cepa experimental de Lutzomyia longipalpis (Diptera: Psychodidae) a tres insecticidas de uso en salud pública en Colombia
}

\author{
Catalina Marceló, Olga Lucía Cabrera, Erika Santamaría \\ Grupo de Entomología, Instituto Nacional de Salud, Bogotá, D.C., Colombia
}

\begin{abstract}
Introducción. En Colombia, las poblaciones periurbanas de Lutzomyia longipalpis, vector del agente causal de la leishmaniasis visceral en el valle alto y medio del río Magdalena, pueden estar expuestas a aplicaciones de insecticidas con diferentes propósitos, por lo que es importante iniciar una vigilancia de la sensibilidad de esta especie a los insecticidas.

Objetivo. Determinar en una cepa experimental de L. longipalpis los indicadores de sensibilidad (concentraciones y tiempos diagnósticos) para tres insecticidas de uso común en salud pública: el malatión, la deltametrina y la lambdacialotrina.

Materiales y métodos. Se utilizó el método propuesto por los Centers for Disease Control and Prevention. Grupos de 10 a 15 hembras se expusieron a diferentes concentraciones de los insecticidas, utilizando botellas de vidrio de $250 \mathrm{ml}$ como cámaras de prueba. La mortalidad se registró cada cinco minutos durante una hora. Se calcularon las concentraciones y los tiempos diagnósticos para cada insecticida.

Resultados. En la cepa evaluada de L. longipalpis, las concentraciones y los tiempos diagnósticos fueron de $75 \mu \mathrm{g} / \mathrm{ml}$ y 25 minutos para el malatión, de $10 \mu \mathrm{g} / \mathrm{ml}$ y 35 minutos para la deltametrina y de $15 \mu \mathrm{g} / \mathrm{ml}$ y 30 minutos para la lambdacialotrina.

Conclusión. Se estableció la línea base de la mortalidad frente a tres insecticidas en una cepa sensible del vector L. longipalpis. Estos indicadores permitirán establecer comparaciones con poblaciones del vector expuestas regular o esporádicamente a medidas de control químico y detectar, así, cambios en la sensibilidad de esta especie a los insecticidas.
\end{abstract}

Palabras clave: leishmaniasis, Lutzomyia, control de insectos, resistencia a insecticidas.

doi: http://dx.doi.org/10.7705/biomedica.v34i4.2233

Discriminating concentrations for three insecticides used in public health in a Lutzomyia longipalpis experimental strain from Colombia

Introduction: In Colombia, periurban populations of Lutzomyia longipalpis, vector of the causative agent of visceral leishmaniasis in the upper and middle valley of the Magdalena River, may be exposed to insecticide applications with different purposes. Thus, it is important to begin a susceptibility surveillance of this species to insecticides.

Objective: To determine indicators of susceptibility to three insecticides habitually used in public health, such as malathion, deltamethrin and lambda-cyhalothrin in an experimental strain of L. longipalpis.

Materials and methods: We used the method proposed by the Centers for Disease Control and Prevention. Groups of 10 to 15 unfed females were exposed to different concentrations of the insecticides using $250 \mathrm{ml}$ glass bottles as test chambers. Mortality was registered every five minutes for an hour. Diagnostic concentrations and lethal times for each insecticide were calculated.

Results: In the evaluated L. longipalpis strain, the diagnostic concentrations and times were $75 \mu \mathrm{g} /$ $\mathrm{ml}$ and 25 minutes for malathion, $10 \mu \mathrm{g} / \mathrm{ml}$ and 35 minutes for deltamethrin, and $15 \mu \mathrm{g} / \mathrm{ml}$ during 30 minutes for lambda-cyhalothrin.

Conclusions: Baseline data over mortality response to three insecticides were established in a susceptibility strain of $L$. longipalpis vector. These indicators will allow establishing comparisons with populations of $L$. longipalpis exposed regularly or sporadically to chemical control measures to detect changes in their resistance to these insecticides.

Key words: Leishmaniasis, Lutzomyia, insect control, insecticide resistance.

doi: http://dx.doi.org/10.7705/biomedica.v34i4.2233

Contribución de los autores:

Catalina Marceló y Olga Lucía Cabrera: diseño metodológico, realización de los ensayos biológicos y apoyo en la elaboración del manuscrito

Erika Santamaría: conducción del estudio, preparación y corrección del manuscrito

Todos los autores revisaron y aprobaron la versión final del manuscrito. 
Lutzomyia longipalpis (Diptera: Psychodidae) es el principal vector de Leishmania infantum, agente etiológico de la leishmaniasis visceral de amplia distribución en las Américas, desde el norte de México hasta el sur de Argentina $(1,2)$. En Colombia, esta especie se ha reportado en nueve departamentos: Huila, Tolima, Cundinamarca, Santander, Caldas, Antioquia, Córdoba, Sucre y La Guajira (3-6). Sin embargo, solo se ha asociado con brotes de leishmaniasis visceral en los primeros cuatro departamentos (7-10). El brote más reciente de la enfermedad ocurrió en el 2012, en el área periurbana de la ciudad de Neiva (Huila) con siete casos confirmados (7).

Lutzomyia longipalpis es reconocida por su habilidad para adaptarse a condiciones urbanas y periurbanas $(10,11)$, y por sus hábitos de reposo temporal en áreas peridomésticas, especialmente en las paredes de los cobertizos para animales (12). Estas características ecológicas hacen que el rociamiento de las superficies del entorno de la vivienda con insecticidas de acción residual, sea una medida de control vectorial potencialmente efectiva $y$, aunque las evaluaciones son escasas, se han obtenido resultados positivos en Brasil y Venezuela $(13,14)$.

En Colombia, las operaciones ordinarias de control vectorial para las leishmaniasis, se hacen bajo condiciones de epidemia o de brotes y en situaciones que indiquen transmisión en el intradomicilio y peridomicilio, y se limitan al rociamiento de las viviendas con insecticidas y a la utilización de toldillos tratados con insecticidas. Sin embargo, no se han documentado las evaluaciones del impacto entomológico o epidemiológico de estas intervenciones (Pardo R. El control vectorial de la leishmaniasis en Colombia: experiencias y retos para el futuro. Congreso de la Sociedad Colombiana de Entomología, Bogotá, 30 de junio a 2 de julio de 2010).

Para el control vectorial específico de la leishmaniasis visceral transmitida por L. longipalpis en el país, y ante la confirmación de, por lo menos, un caso de la enfermedad, se han aplicado insecticidas residuales en las paredes externas de las viviendas, así como en los maderos y travesaños

\section{Correspondencia:}

Erika Santamaría, Grupo de Entomología, Instituto Nacional de Salud, Bogotá, D.C., Colombia

Teléfono: (571) 2207700 , extensión 1278

esantamaria@ins.gov.co

Recibido: 31/12/13; aceptado: 18/07/14 de los cobertizos para animales cercanos a las viviendas, siempre y cuando haya evidencia previa del reposo del vector en estas superficies en el área específica intervenida (15). Esta medida se toma con el fin de disminuir la abundancia del vector y reducir la exposición de las personas a las picaduras infecciosas de los flebótomos (16).

Otra medida complementaria del rociamiento cuando hay brotes, ha sido la distribución entre la población de toldillos tratados con insecticidas de larga duración, como se hizo durante el brote de leishmaniasis visceral en Neiva en 2012 (no se han publicado los datos; Secretaría de Salud del Huila). No obstante, la eficacia de los toldillos tratados con insecticidas para el control de vectores con hábitos crepusculares y baja endofagia, como es el caso de L. longipalpis, puede ser discutible. En un estudio de tipo cruzado llevado a cabo en Brasil, Courteney, et al. (17), encontraron una reducción del $80 \%$ en las hembras de L. longipalpis que se hallaron posadas sobre atrayente humano dentro de los toldillos tratados con deltametrina, en comparación con las halladas en los toldillos sin tratar. Como parte de las acciones mencionadas de control vectorial, es importante conocer el estado actual de la sensibilidad a los insecticidas de $L$. longipalpis en poblaciones sujetas a control químico.

Además, algunas poblaciones periurbanas de $L$. longipalpis en nuestro país pueden estar expuestas a las aspersiones periódicas de insecticidas realizadas en el marco de los programas de control vectorial del dengue (datos del Grupo de Entomología del Instituto Nacional de Salud), lo cual puede ocasionar el desarrollo de resistencia a los insecticidas en ellas. Por esta razón, es necesario establecer los indicadores de sensibilidad a insecticidas de $L$. longipalpis, principalmente en áreas de transmisión de leishmaniasis visceral, y hacerles seguimiento en el tiempo.

Aunque se considera que los flebótomos del Nuevo Mundo siguen siendo sensibles a los insecticidas usados para su control (18), en poblaciones de L. longipalpis de países limítrofes con Colombia existen datos que indican una disminución de la sensibilidad a los insecticidas, la cual podría derivar en resistencia. En Venezuela, Mazzari, et al. (19), detectaron mayores tiempos letales medios $\left(T L_{50}\right)$ en los insecticidas metilpirimifos, fenitrotión y permetrina en una población de $L$. longipalpis expuesta a insecticidas con propósitos agrícolas, comparada con una cepa sensible de 
laboratorio. En Brasil se registraron valores de $\mathrm{TL}_{50}$ para los piretroides permetrina y deltametrina, significativamente más altos en una población de $L$. longipalpis expuesta frecuentemente a piretroides para el control de la leishmaniasis visceral que los encontrados en una población de L. longipalpis geográficamente distante y sin evidencia de exposición a insecticidas, lo que se confirmó con análisis bioquímicos de la actividad de las enzimas de desintoxicación de insecticidas (20).

Lo anterior demuestra la importancia de iniciar y mantener en el país una vigilancia de la sensibilidad de las poblaciones de L. longipalpis a los insecticidas usados en el control vectorial.

El objetivo de este trabajo fue determinar en una cepa experimental de L. longipalpis, las concentraciones y los tiempos diagnósticos para tres insecticidas de uso común en salud pública: el malatión, la deltametrina y la lambdacialotrina. El malatión se usa comúnmente en el rociamiento de las paredes de las viviendas para el control de Aedes aegypti (21) en áreas urbanas y periurbanas, donde puede coincidir con la distribución de $L$. longipalpis. La deltametrina y la lambdacialotrina se recomiendan para el tratamiento residual de viviendas en el control de flebótomos (15) y son los ingredientes activos de los insecticidas de larga duración con los que se tratan los toldillos $(22,23)$.

La obtención de la línea basal de los indicadores de sensibilidad (concentraciones y tiempos diagnósticos) de L. longipalpis al malatión, la deltametrina y la lambdacialotrina, permitirá hacer comparaciones con poblaciones silvestres de esta especie sometidas regular o esporádicamente a control químico con estos insecticidas.

\section{Materiales y métodos}

Se utilizaron insectos de una colonia cerrada de L. longipalpis establecida en el Grupo de Entomología del Instituto Nacional de Salud desde 1995 con ejemplares procedentes de la vereda $\mathrm{EI}$ Callejón, municipio de Ricaurte, Cundinamarca, según el procedimiento de Modi y Tesh (24), la cual no había sido expuesta a insecticidas. Para los ensayos biológicos se emplearon grupos de 10 a 15 hembras sin alimentación sanguínea, de 1 a 3 días de edad, con suministro previo de agua y solución azucarada ad libitum.

Se utilizó el método simplificado propuesto por los Centers for Disease Control and Prevention (CDC) para determinar la sensibilidad a insecticidas de mosquitos adultos $(25,26)$. La superficie interna y las tapas de botellas de vidrio Wheaton ${ }^{\circledR}$ de 250 $\mathrm{ml}$, se trataron con $1 \mathrm{ml}$ de la solución insecticida de acuerdo con el método mencionado, pero con las siguientes modificaciones: 1) como solvente se empleó alcohol etílico absoluto Merck ${ }^{\circledR}$ en lugar de acetona, debido a que en estudios previos las botellas tratadas únicamente con acetona causaron postración en los flebótomos $(27,28)$, y 2) el tiempo de impregnación total de las botellas con la solución insecticida fue de diez minutos, la mitad de este tiempo con la tapa puesta y la otra mitad sin la tapa.

Los insecticidas bajo prueba fueron el organofosforado malatión y los piretroides deltametrina y lambdacialotrina. Las concentraciones evaluadas fueron: de 50, 75, 100 y $150 \mu \mathrm{g} / \mathrm{ml}$ para el malatión; de $5,7,10,25$ y $45 \mu \mathrm{g} / \mathrm{ml}$ para la deltametrina; $y$ de $3,5,10,15,25$ y $50 \mu \mathrm{g} / \mathrm{ml}$ para la lambdacialotrina. Dichas concentraciones se establecieron con base en ensayos preliminares, según las recomendaciones de los CDC (26) y tomando como referencia las concentraciones establecidas en estudios previos con flebótomos $(27,29)$.

Después de introducir las hembras en la botella, se registró el número de hembras muertas cada cinco minutos hasta completar una hora. Se consideró como muerta la hembra derribada que no presentaba actividad después de un movimiento suave de la botella. Para evitar escapes de los insectos en el momento de introducir el grupo de hembras a la botella, se colocó una sección de muselina de $7 \times 7 \mathrm{~cm}$ con un agujero de $1 \mathrm{~cm}$ de diámetro en el centro, la cual se ajustó al cuello de la botella con una banda de caucho. Cada conjunto de pruebas incluyó siete botellas con dos concentraciones diferentes de cada insecticida, más una botella de control (tratada con $1 \mathrm{ml}$ del solvente). Cada concentración se repitió 12 veces. Durante los ensayos, la temperatura osciló entre 23 y $25^{\circ} \mathrm{C}$, y la humedad relativa, entre 60 y $70 \%$.

Se hicieron las gráficas del tiempo de exposición comparado con la mortalidad y se estimó para cada insecticida la concentración diagnóstica, entendida como la mínima concentración con la cual se obtiene $100 \%$ de mortalidad de los insectos sensibles, y el tiempo diagnóstico, o umbral de resistencia en el cual se alcanza dicha mortalidad $(25,26)$.

\section{Resultados}

Se usaron en total 2.308 hembras de $L$. longipalpis en los ensayos, 628 con el insecticida organofosforado y 1.680 con los piretroides. 
En la figura 1 se presenta el porcentaje de mortalidad de las hembras con relación al tiempo de observación en las diferentes concentraciones de malatión, deltametrina y lambdacialotrina, así como el tiempo diagnóstico. En el cuadro 1 se resumen los indicadores de sensibilidad de L. longipalpis para los tres insecticidas, los cuales constituyen el punto de referencia para las comparaciones con poblaciones silvestres de este vector.

En las pruebas con los insecticidas deltametrina y lambdacialotrina, al finalizar el tiempo de exposición y retirar las hembras de L. longipalpis, se
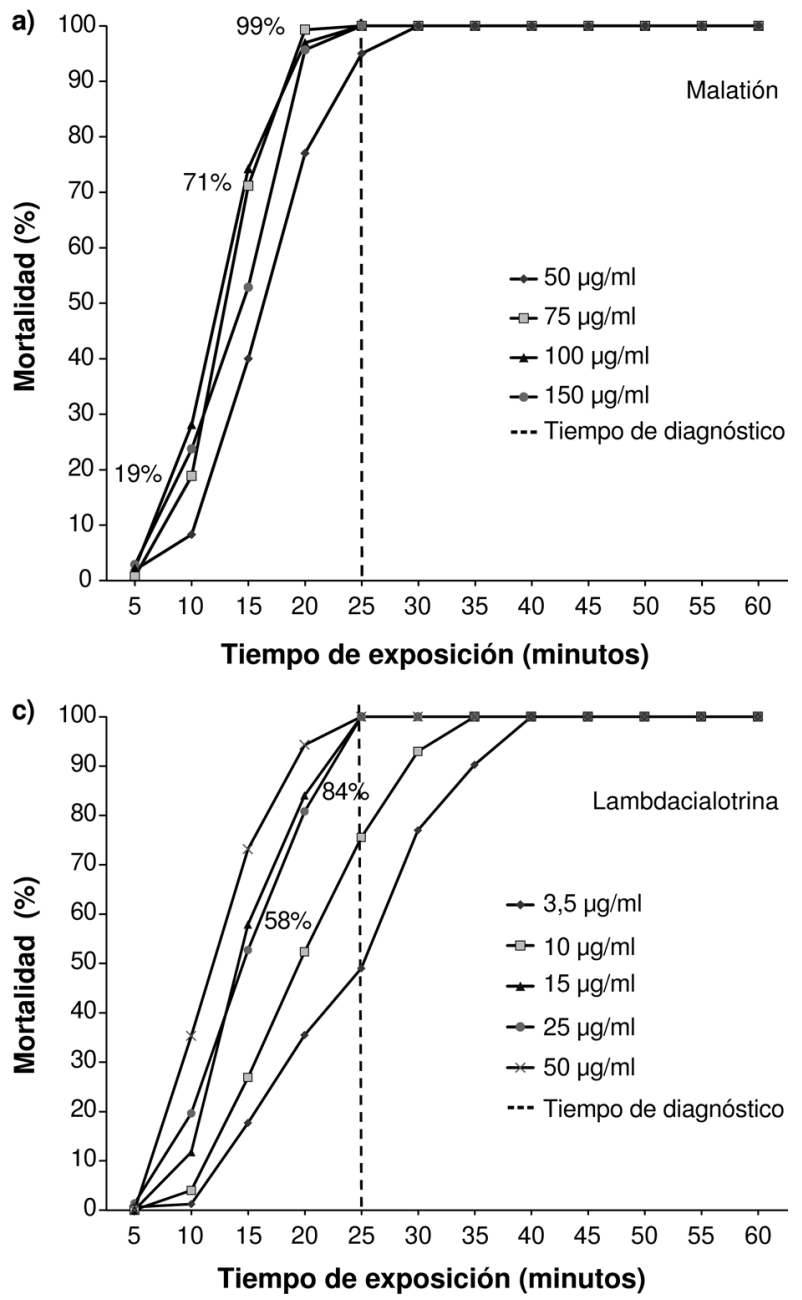

observó un gran número de patas desprendidas en la superficie de la botella, efecto que se ha observado en otros estudios tras la exposición de esta especie a los piretroides $(20,30)$.

\section{Discusión}

Los reportes de sensibilidad de L. longipalpis a insecticidas provienen de Brasil $(20,31,32)$ y Venezuela (19), pero se recomienda llevar a cabo estudios a nivel local porque pueden existir variaciones entre especies en cuanto a la sensibilidad a los insecticidas, que dependen del historial de su

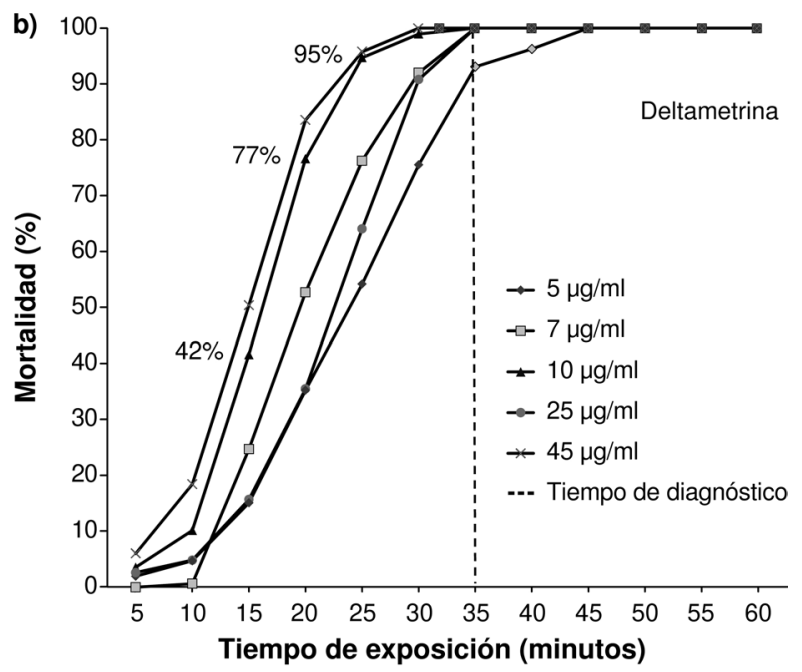

Figura 1. Mortalidad de hembras de Lutzomyia longipalpis con relación al tiempo de exposición para varias concentraciones de: a) malatión, b) deltametrina y c) lambdacialotrina, empleando el método de los CDC. Los resultados se muestran como porcentajes de mortalidad del número total de hembras expuestas.

Cuadro 1. Indicadores de sensibilidad a tres insecticidas en una cepa experimental de Lutzomyia longipalpis obtenidos con el método de los Centers for Disease Control and Prevention

\begin{tabular}{lcccc}
\hline Insecticida & $\begin{array}{c}\text { Concentración } \\
\text { diagnóstica }(\boldsymbol{\mu g} / \mathbf{m l})\end{array}$ & $\begin{array}{c}\text { Tiempo diagnóstico } \\
\text { (minutos) }\end{array}$ & $\begin{array}{c}\text { Tiempo letal 50 } \\
\text { (minutos) }\end{array}$ & $\begin{array}{c}\text { Tiempo letal 95 } \\
\text { (minutos) }\end{array}$ \\
\hline Malatión & 75 & 25 & 12 & 23 \\
Deltametrina & 10 & 35 & 15 & 35 \\
Lambdacialotrina & 15 & 30 & 14 & 27 \\
\hline
\end{tabular}


exposición a las medidas de control químico $(25,26)$. En los estudios previos se ha utilizado el método de la Organización Mundial de la Salud (OMS), principalmente para determinar los tiempos letales con una concentración fija, registrando la mortalidad 24 horas después de la exposición, por lo que esos datos no pueden compararse directamente con los del presente estudio.

En Colombia no se habían reportado antes indicadores de sensibilidad al malatión y la deltametrina para L. longipalpis. En cuanto a la lambdacialotrina, hay un estudio preliminar de estandarización de la metodología de los CDC, en el que se estimó una concentración diagnóstica inicial de $10 \mu \mathrm{g} / \mathrm{ml}$ (29); sin embargo, solo se probaron tres concentraciones del insecticida, lo cual dificultó la identificación precisa del tiempo de diagnóstico.

En cuanto a otras especies de flebótomos de importancia en salud pública en el país, solo se ha determinado la sensibilidad a insecticidas (deltametrina y lambdacialotrina) en $L$. evansi, vector de $L$. infantum en la Costa Atlántica (27), para lo cual se aplicó la metodología de los CDC, usando ejemplares silvestres de la especie y un tiempo total de exposición de 80 minutos. Al comparar las concentraciones y tiempos diagnósticos de $L$. evansi (27) con los obtenidos para L. longipalpis en el presente estudio, se encontró que eran más altos en este último vector, tanto para la deltametrina $(7 \mu \mathrm{g} / \mathrm{ml}$ y 10 minutos en L. evansi $V s$. $10 \mu \mathrm{g} / \mathrm{ml}$ y 35 minutos en $L$. longipalpis), como para la lambdacialotrina $(3,5 \mu \mathrm{g} / \mathrm{ml}$ y 10 minutos en L. evansi Vs. $15 \mathrm{ug} /$ $\mathrm{ml}$ y 30 minutos en L. longipalpis). Lo anterior puede indicar diferencias en la tolerancia fisiológica natural de las distintas especies de flebótomos a los insecticidas y, probablemente, involucre variables como el tamaño de la especie y la actividad de sus enzimas de desintoxicación.

Entre las ventajas del método de los CDC para determinar la sensibilidad a insecticidas en flebótomos frente al método de la OMS (33), puede mencionarse la posibilidad de probar cualquier concentración y no solo las concentraciones diagnósticas de los papeles impregnados usados en el método de la OMS, que son adecuadas para mosquitos, mas no para flebótomos. Por otra parte, la botella de vidrio empleada como cámara de exposición, comparada con el recipiente de exposición de la OMS, tiene toda la superficie interior tratada con el insecticida, minimiza los escapes de los flebótomos y permite observar su comportamiento mientras están expuestos al insecticida.
En conclusión, se presentan las concentraciones y los tiempos diagnósticos del malatión, la deltametrina y la lambdacialotrina en una cepa sensible de L. longipalpis, usando el método de los CDC. Se espera que estos datos de base puedan ser empleados en evaluaciones de sensibilidad a estos mismos insecticidas en poblaciones de L. longipalpis expuestas a control químico en áreas de transmisión de la leishmaniasis visceral en Colombia.

\section{Agradecimientos}

A Ligia Lugo, Liliana Santacoloma y Gabriela Rey del Grupo de Entomología del Instituto Nacional de Salud, por la provisión de los ingredientes activos de los insecticidas y su asesoría en el estudio; a Marco Fidel Suárez, por el mantenimiento de la cepa experimental de L. longipalpis y su apoyo durante las pruebas.

\section{Conflicto de intereses}

Los autores declaran que no existe conflicto de intereses.

\section{Financiación}

Instituto Nacional de Salud y Colciencias (proyecto de investigación, código 124349326165, Programa Jóvenes Investigadores, 2011, y Programa de Doctorados Nacionales, Generación del Bicentenario).

\section{Referencias}

1. Young DG, Duncan MA. Guide to the identification and geographic distribution of Lutzomyia sand flies in México. The West Indies, Central and South America (Diptera: Psychodidae). Mem Am Entomol Inst. 1994;54:1-881.

2. Lainson R, Elizabeth R. Lutzomyia longipalpis and the eco-epidemiology of American visceral leishmaniasis, with particular reference to Brazil -a review. Mem Inst Oswaldo Cruz. 2005;100:811-27. http://dx.doi.org/10.1590/S007402762005000800001

3. Montoya-Lerma J, Ferro C. Flebótomos (Diptera: Psychodidae) de Colombia. En: Amat G, Andrade MG, Fernández F, editores. Insectos de Colombia. Colección Jorge Álvarez Lleras, No. 13, Academia Colombiana de Ciencias Exactas, Físicas y Naturales. Santafé de Bogotá: Centro Editorial Javeriano; 1999. p. 211-45.

4. Bejarano EE. Lista actualizada de los psicódidos (Diptera: Psychodidae) de Colombia. Folia Entomol Mex. 2006;45: 47-56.

5. González C, Cabrera OL, Munstermann LE, Ferro C. Distribución de los vectores de Leishmania infantum (Kinetoplastida: Trypanosomatidae) en Colombia. Biomédica. 2006;26(Supl.1):64-72. http://dx.doi.org/10.7705/biomedica. v26i1.1501

6. Acosta LA, Mondragón-Shem K, Vergara D, VélezMira A, Cadena H, Carrillo-Bonilla L. Ampliación de la distribución de Lutzomyia longipalpis (Lutz \& Neiva, 1912) 
(Diptera: Psychodidae) en el departamento de Caldas: potencial aumento del riesgo de leishmaniasis visceral. Biomédica. 2013;33:319-25. http://dx.doi.org/10.7705/ biomedica.v33i2.762

7. Gómez-Romero SE, Zambrano P. Informe del evento leishmaniasis hasta el XII periodo epidemiológico del año 2012. Bogotá, D.C.: INS; 2012.

8. Corredor A, Álvarez CA, Agudelo CA, Bueno M, López MC, Cáceres E, et al. Prevalence of Trypanosoma cruzi and Leishmania chagasi infection and risk factors in a Colombian indigenous population. Rev Inst Med Trop. 1999;41:229-34. http://dx.doi.org/10.1590/S0036-46651999000400005

9. Morrison AC, Ferro C, Morales A, Tesh R, Wilson ML. Dispersal of the sand fly Lutzomyia longipalpis (Diptera: Psychodidae) at an endemic focus of visceral leishmaniasis in Colombia. J Med Entomol. 1993;30:427-35.

10. Flórez M, Martínez JP, Gutiérrez R, Luna KP, Serrano VH, Ferro C, et al. Lutzomyia longipalpis (Diptera: Psychodidae) en un foco suburbano de leishmaniosis visceral en el Cañón del Chicamocha en Santander, Colombia. Biomédica. 2006; 26(Supl.1):109-20. http://dx.doi.org/10.7705/biomedica.v26i1. 1505

11. Vivero RJ, Bejarano EE, Pérez-Doria A, Flórez F, Estrada LG, Torres C, et al. Nuevos registros de flebotomíneos (Diptera: Psychodidae), con el hallazgo de Lutzomyia longipalpis (Lutz \& Neiva, 1912), en los alrededores de la ciudad de Sincelejo, Colombia. Biota Neotrop. 2009;9:27780. http://dx.doi.org/10.1590/S1676-06032009000400031

12. Morrison AC, Ferro C, Pardo R, Torres M, Wilson M, Tesh R. Nocturnal activity patterns of Lutzomyia longipalpis at an endemic focus of visceral leishmaniasis in Colombia. J Med Entomol. 1995;32:605-17.

13. Kelly DW, Mustafa Z, Dye C. Differential application of lambda-cyhalothrin to control the sandfly Lutzomyia longipalpis. Med Vet Entomol. 1997;11:13-24. http://dx.doi. org/10.1111/j.1365-2915.1997.tb00285.x

14. Feliciangeli MD, Mazarri MB, San Blas S, Zerpa 0 . Control trial of Lutzomyia longipalpis s.l. in the Island of Margarita, Venezuela. Trop Med Int Health. 2003;8:1131-6. http://dx.doi.org/10.1046/j.1360-2276.2003.01137.x

15. Ministerio de la Protección Social, Instituto Nacional de Salud, Organización Panamericana de la Salud. Gestión para la vigilancia entomológica y control de la transmisión de leishmaniasis. Guía técnica. 2012. Fecha de consulta: 3 de diciembre de 2013. Disponible en: http://www. ins.gov.co/temas-de interes/Leishmaniasis\%20viceral/03\%20 Vigilancia\%20Entomo\%20Leishmaniasis.pdf.

16. Molyneux DH. Control. En: Cox F, editor. Modern parasitology, a textbook of parasitology. Cambridge: Blackwell Science; 1993. p. 243-63.

17. Courteney O, Gillingwater K, Gomes PA, Garcez LM, Davies CR. Deltamethrin-impregnated bednets reduce human landing rates of sandfly vector Lutzomyia longipalpis in Amazon households. Med Vet Entomol. 2007;21:168-76. http://dx.doi.org/10.1111/j.1365-2915.2007.00678.x

18. Alexander B, Maroli M. Control of phlebotomine sandflies. Med Vet Entomol. 2003;17:1-18. http://dx.doi.org/10.1046/ j.1365-2915.2003.00420.x
19. Mazzarri MB, Feliciangeli MD, Maroli M, Hernández A, Bravo A. Susceptibility of Lutzomyia longipalpis (Diptera: Psychodidae) to selected insecticides in an endemic focus of visceral leishmaniasis in Venezuela. J Am Mosq Control Assoc. 1997;13:335-41.

20. Alexander B, Barros VC, De Souza SF, Barros SS, Teodoro LP, Soares ZR, et al. Susceptibility to chemical insecticides of two Brazilian populations of the visceral leishmaniasis vector Lutzomyia longipalpis (Diptera: Psychodidae). Trop Med Int Health.2009;14:272-7. http://dx. doi.org10.1111/ j.1365-3156.2009.02371.x

21. Ministerio de la Protección Social, Instituto Nacional de Salud, Organización Panamericana de la Salud. Gestión para la vigilancia entomológica y control de la transmisión de dengue. Guía técnica. 2011. Fecha de consulta: 9 de diciembre de 2013. Disponible en: http://www.ins.gov.co/ temas-de-interes/Dengue/03\%20Vigilancia\%20entomo\%20 dengue.pdf.

22. World Health Organization Pesticide Evaluation Scheme (WHOPES). Report of the 12th WHOPES Working Group meeting -Review of Bioflash ${ }^{\circledR}$ GR, Permanet ${ }^{\circledR}$ 2.0, Permanet巴 3.0, Permanet $\AA$ 2.5, Lambda-cyhalothrin LN. December 2008. WHO/HTM/NTD/WHOPES/2009.1. Geneva: World Health Organization; 2008. 120 p.

23. World Health Organization Pesticide Evaluation Scheme (WHOPES). Report of the 11th WHOPES Working Group meeting - Review of Spinosad 7.48\% DT, Netprotect ${ }^{\circledR}$, Duranet $^{\circledR}$, Dawaplus ${ }^{\circledR}$, Icon ${ }^{\circledR} \operatorname{maxx}$. December 2007. WHO/ HTM/NTD/WHOPES/2008.1. Geneva: World Health Organization; 2007. $105 \mathrm{p}$

24. Modi GB, Tesh RB. A simple technique for mass rearing Lutzomyia longipalpis and Phlebotomus papatasi (Diptera: Psychodidae) in the laboratory. J Med Entomol. 1983;20: 568-9.

25. Brogdon WG, McAllister JC. Simplification of adult mosquito bioassays through use of time-mortality determinations in glass bottles. J Am Mosq Control Assoc.1998;14:159-64.

26. Centers for Disease Control and Prevention. Guideline for evaluating insecticide resistance in vectors using the CDC bottle bioassay. U. S. Department of Health and Human Services. 2011. Fecha de consulta: 9 de noviembre de 2013. Disponible en: http://www.cdc.gov/malaria/resources/ pdf/fsp/ir_manual/ir_cdc_bioassay_en.pdf.

27. Henríquez C, Pereira Y, Cochero S, Bejarano EE. Dosis diagnóstica y umbral de resistencia de Lutzomyia evansi (Diptera: Psychodidae), a dos insecticidas utilizados en salud pública en Colombia: deltametrina y lambdacihalotrina. Rev Soc Entomol Argent. 2009;68:287-94.

28. Marceló C. Determinación de la susceptibilidad de Lutzomyia longipalpis (Lutz \& Neiva 1912), vector de Leishmania infantum (Nicolle 1908), a insecticidas de uso en salud pública. Tesis de Licenciatura en Biología. Bogotá, D.C.: Universidad Distrital "Francisco José de Caldas"; 2010. 91 p.

29. Santamaría E, Munstermann L, Ferro C. Aproximación al método CDC para determinar susceptibilidad a insecticidas en vectores de leishmaniasis. Biomédica. 2003;23:115-21. http://dx.doi.org/10.7705/biomedica.v23i1.1202

30. Bray DP, Alves GB, Dorval ME, Brazil RP, Hamilton JG. Synthetic sex pheromone attracts the leishmaniasis vector 
Lutzomyia longipalpis to experimental chicken sheds treated with insecticide. Parasit Vectors. 2010;3:16. http://dx.doi. org/10.1186/1756-3305-3-16

31. Falcao AR, Pinto CT, Gontijo CM. Susceptibility of Lutzomyia longipalpis to deltamethrin. Mem Inst Oswaldo Cruz. 1988;83:395-6. http://dx.doi.org/10.1590/S0074-02761988 000300020
32. Oliveira F, Melo M. The chemical control of vectors of leishmaniasis. Mem Inst Oswaldo Cruz.1994;89:461-2. http://dx.doi.org/10.1590/S0074-02761994000300031

33. Organización Mundial de la Salud. Resistencia a los insecticidas y lucha contra vectores. Serie de informes técnicos N. 443. $17^{\circ}$ informe del Comité de expertos de la OMS en insecticidas. Ginebra: OMS; 1970. 106 p. 